\title{
Experimental methods applied in a study of stall flutter in an axial flow fan
}

\author{
John D. Gill ${ }^{\mathrm{a}}$, Vincent R. Capece ${ }^{\mathrm{a}, *}$ and Ronald B. Fost ${ }^{\mathrm{b}}$ \\ ${ }^{a}$ Department of Mechanical Engineering, University of Kentucky, USA \\ ${ }^{\mathrm{b}}$ Battelle Memorial Institute, Compressor Research Facility, WPAFB, Dayton, OH, USA
}

Received 6 May 2003

Revised 18 November 2003

\begin{abstract}
Flutter testing is an integral part of aircraft gas turbine engine development. In typical flutter testing blade mounted sensors in the form of strain gages and casing mounted sensors in the form of light probes (NSMS) are used. Casing mounted sensors have the advantage of being non-intrusive and can detect the vibratory response of each rotating blade. Other types of casing mounted sensors can also be used to detect flutter of rotating blades. In this investigation casing mounted high frequency response pressure transducers are used to characterize the part-speed stall flutter response of a single stage unshrouded axial-flow fan. These dynamic pressure transducers are evenly spaced around the circumference at a constant axial location upstream of the fan blade leading edge plane. The pre-recorded experimental data at $70 \%$ corrected speed is analyzed for the case where the fan is back-pressured into the stall flutter zone. The experimental data is analyzed using two probe and multi-probe techniques. The analysis techniques for each method are presented. Results from these two analysis methods indicate that flutter occurred at a frequency of $411 \mathrm{~Hz}$ with a dominant nodal diameter of 2 . The multi-probe analysis technique is a valuable method that can be used to investigate the initiation of flutter in turbomachines.
\end{abstract}

\section{Introduction}

The necessity to understand the fundamental problems associated with the design and operation of turbomachines becomes increasingly important as engineers strive to enhance their performance. One of the challenges often encountered in the design and operation of fans and compressors for both commercial and military aircraft gas turbine engines is flutter. Current design trends of thinner blades and higher pressure ratio per stage make fan and compressor blades more susceptible to flutter. When flutter is encountered in the design and development of a fan or compressor, an expensive redesign and retest program results.

As schematically depicted in Fig. 1 on a compressor performance map, there are many different types of flutter that can be encountered in the fan and compressor sections of the engine. Supersonic unstalled flutter occurs near the design point where the incidence angles are nominal and the Mach number relative to the blade is supersonic. As the pressure ratio increases at high corrected speeds supersonic stall flutter is encountered near the stall line. At off-design conditions where the corrected speeds are low, choke flutter can be found in the aft stages of high pressure compressors for negative incidence angles and subsonic/transonic stall flutter in the fan or front stages of the high pressure compressor.

The development of analyses to predict flutter is an area of research interest. Due to the complexity of the flow field and the fluid/structure interaction phenomena, accurate prediction of flutter at all operating conditions has remained elusive. Subsonic/transonic stall flutter, in particular, is difficult to predict. This is caused by the high incidence angles, which can be $10 \mathrm{deg}$. or higher. For these incidence angles the flow separates and viscous effects

*Corresponding author. E-mail: vcapece@engr.uky.edu. 


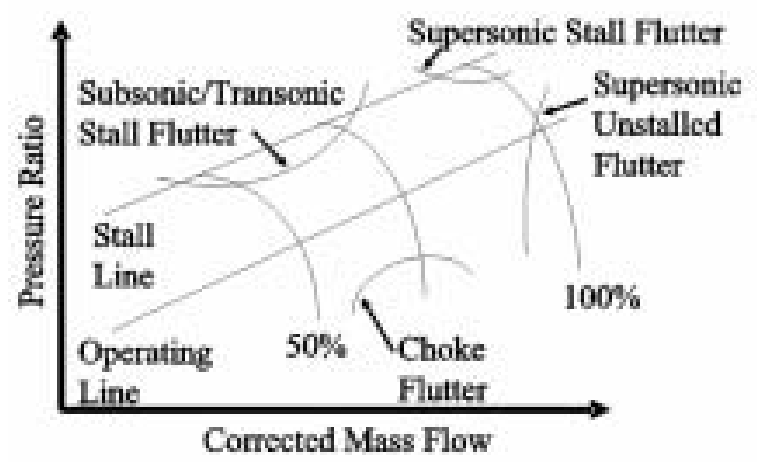

Fig. 1. Typical performance map of a fan or compressor showing the possible flutter regimes.

are important. Consequently, the Navier-Stokes equations need to be considered. Unsteady Reynolds Averaged Navier-Stokes (RANS) models (e.g. $[1,11,13,15,16])$ have been developed to account for viscous effects. The calculation of separated flow phenomena is a general weakness of computational fluid dynamic predictions.

Hence, flutter testing is an important component in the design and development of fan and compressor blades. To measure the dynamic response of the blades during flutter rotating frame and/or stationary frame sensors can be employed. Strain gages are typically used as the rotating frame sensors. These sensors are bonded to the blades with the lead wires routed through a slip-ring to the stationary frame for display of signals during testing. Additionally, the strain gage signals are recorded for post-test analysis. The advantage of strain gage sensors is the direct assessment of the stress during flutter. However, there are some disadvantages to the use of strain gages.

The strain gage sensors and lead wires, albeit thin, disrupt the flow over the surface of the blade, which has an effect on the blade aerodynamic performance. Moreover, the sensors and lead wires can influence the vibration characteristics of the blades. Due to this negative impact on the blade performance and structural dynamic characteristics it is desirable to minimize the number of strain gages and still provide adequate coverage of the possible vibration modes of the blade (see for example Mignolet and Choi [9]). A major concern with this approach is not having a strain gage on a blade that has the highest stress response. Furthermore, due to the harsh engine operating environment the failure rates of the strain gages during testing can be high.

To overcome some of these difficulties blade vibration measurements using stationary frame sensors has been pursued. Stationary sensors mounted in the casing of the fan or compressor have the advantage of being non-intrusive. High frequency response pressure transducers and optical probes have been used to detect blade vibration from the casing above the rotating blades.

Optical displacement probes have received considerable attention. Optical displacement sensors direct a light beam (typically generated by a laser) onto the tips of the blades as they rotate beneath the sensor. The reflected light from the blade tip is measured using a photomultiplier each time a blade passes by the probe. This measurement is compared to a reference pulse generated for the number of rotor blades to establish the blade instantaneous position. During flutter the time of arrival of the blade would be different than the reference pulse indicating blade vibration. Using the measured deflections the frequency of vibration of the blades in flutter can be quantified. Moreover, from these deflections and knowledge of the dynamic structural response of the blade, the blade stress can be inferred.

There are also challenges concerning the use of the optical displacement system. This system requires optical access that is susceptible to fouling from the inlet flow field. Additionally, the blade tips must have a high degree of reflectivity.

Optical measurements to detect flutter have been used by many investigators. Stargardter [12] used small mirrors mounted on fan blades at different span locations to quantify blade displacement during flutter using the reflections of laser light. Jutras et al. [3] and Kurkov [6] used laser probes to measure the deflection of the fan blades during subsonic/transonic stall flutter. Additionally, Kurkov [7] and Kurkov and Mehmed [8] have used this technique for flutter detection of unducted fans.

Stationary reference frame high frequency response pressure transducers have also been used to determine whether a rotor blade was in flutter. An advantage of this method is that commercially available pressure sensors can be 
used to measure the time-variant pressure during flutter. From the dynamic pressure measurements the vibration frequency and nodal diameter pattern of the rotor blades in flutter can be determined.

Pressure transducers were also used by Jutras et al. [3] to measure the time-variant pressure during subsonic/transonic stall flutter. Kurkov [4] used pressure and displacement measurements to determine the unsteady aerodynamic work during flutter.

Typically, flutter detection using either pressure transducers or optical sensors employs two sensors mounted in the casing above the rotor. By using two sensors at the same axial location with different circumferential locations, the flutter frequency and nodal diameter (interblade phase angle) can be quantified.

In this paper the flutter characteristics of an unshrouded fan blade are quantified using casing mounted high response pressure transducers. The configuration employed in this investigation consists of multiple high frequency response pressure transducers mounted evenly around the casing circumference in the tip region of the rotor blades. This configuration allows the data analysis to be conducted as if the data were collected in the rotating reference frame. The data analysis techniques are developed mathematically for the two probe and multiprobe methods.

\section{Experiment}

This experimental program was conducted in the Compressor Research Facility (CRF), which is one of four test facilities in the Turbine Engine Research Center (TERC) at Wright Patterson Air Force Base. The CRF is capable of experimentally evaluating aerodynamic performance and aeromechanics (flow induced vibrations) of full-scale fans or high pressure compressors used in aircraft gas turbine engines.

Air enters the facility test chamber through a set of inlet filters and pressure regulating valves. These valves allow the inlet pressure to be varied from 2 psia $(13.79 \mathrm{kPa})$ to ambient. From the $20 \mathrm{ft}(6.1 \mathrm{~m})$ diameter by $65 \mathrm{ft}$ $(19.81 \mathrm{~m})$ long test chamber the air flows through flow conditioning elements and enters the fan or compressor. A 30,000 horsepower motor drives a low speed and high speed gearbox that provides accurate speed control for rotational speeds up to $30,000 R P M$. The air exits the fan or compressor through a throttling valve. Downstream of the throttling valve a venturi meter is used to quantify the mass flow rate. Additionally, there is also a second set of ducts available for compression systems that require a bypass stream. This second flow path is also equipped with a separate throttling valve to control the bypass stream flow and venturi meter to measure the mass flow rate.

Conventional instrumentation coupled with a state-of-the-art data acquisition system provide high quality aerodynamic performance data for the fan or compressor component. Temperature and pressure measurements at the inlet and exit of the test article quantify the overall performance of the fan or compressor. To resolve stage performance, pressure and temperature measurements are made at the entrance to each stationary (nonrotating) blade row.

Time-variant data from strain gages and high frequency response pressure transducers are acquired with a wide bandwidth analog data acquisition system (see Fost and Teets [2]). During testing time-variant data is displayed on a combination of oscilloscopes and spectral analyzers. All analog data is also recorded on high fidelity tape for future review and post-test analysis.

This facility has all the capabilities necessary to experimentally investigate stall flutter. In this study stall flutter of a single stage unshrouded axial flow fan was considered. The Honeywell development fan blisk consists of 22 wide-chord fan blades. The blades and disk were fabricated from a single piece of material (integrally bladed disk). This creates low structural damping for the bladed-disk system. Stall flutter was generated by back-pressuring the fan while holding the rotational speed constant. This reduced the mass flow rate and increased the incidence angle moving the fan operating point towards the stall line. Figure 2 shows that stall flutter was encountered over a range of constant speedlines. For this investigation flutter development along the $70 \%$ corrected speedline was considered. Data acquistion was initiated on the stable side of the flutter boundary and continued to the location indicated by the symbol in Fig. 2. At this point the fan was operating in the limit amplitude flutter region.

The time-variant instrumentation consisted of 1) blade mounted high frequency response pressure transducers (Kulite XCQ-062-20A) and strain gages, 2) casing mounted high frequency response pressure transducers located over the rotor blades, and 3) casing mounted laser light probes. The blade mounted strain gages and high frequency response pressure transducers were transferred from the rotating to the stationary reference frame through a forward mounted 100 channel slip-ring (Sanders et al. [10]). 


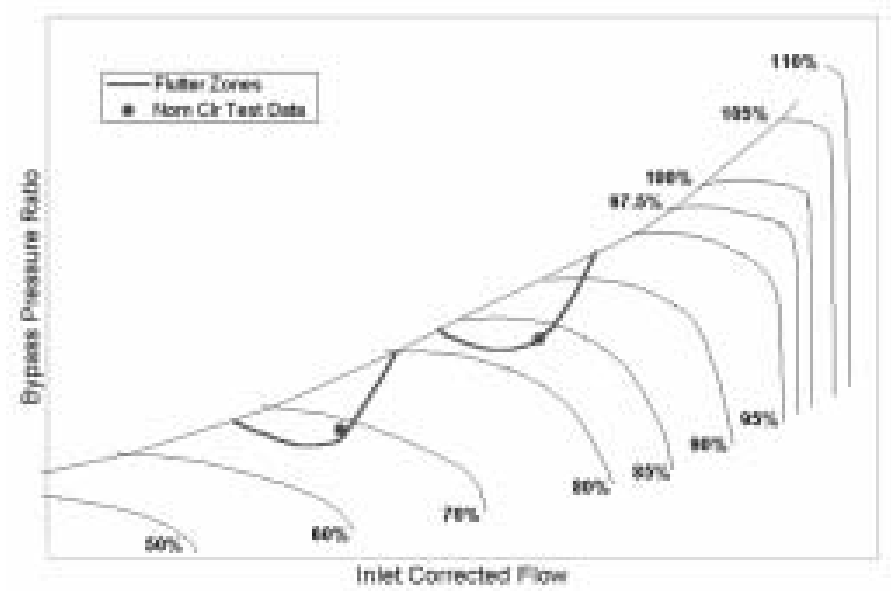

Fig. 2. Performance map showing the flutter boundaries of the unshrouded fan.

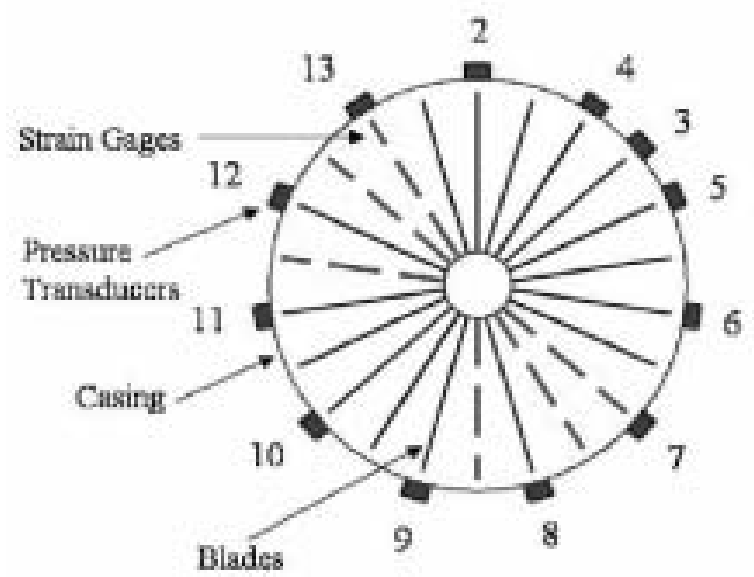

Fig. 3. Schematic diagram of pressure transducer and strain gage locations for the test article.

This investigation focuses on the casing mounted high frequency response pressure transducers near the leading edge of the fan blades, see Fig. 3. Pressure transducers two and four through thirteen were flush mounted upstream of the rotor blade leading edge plane and equally spaced around the circumference of the casing. Transducer three was positioned at the midpoint between transducers four and five. Transducer one, which is not shown in Fig. 3, was mounted in the casing and forward of transducer two. Figure 3 also shows the relative positions of six of the original eight blade mounted strain gages.

\section{Data analysis}

Flutter is a self-induced vibration due to an instability in the bladed disk system of the fan, and is usually associated with one of the system's fundamental modes (bending or torsion) with a frequency close to one of the natural frequencies since the airfoil-to-air density ratio of turbomachine blades is large, unlike aircraft wings. Flutter is comprised of one or more circumferential traveling waves, in the rotating reference frame, each with a distinct nodal diameter. The nodal diameter of the wave is equivalent to the number of cycles the wave makes around the circumference of the rotor, i.e. the spatial frequency of the wave. Each of these waves will have an associated traveling pressure wave. Due to the periodicity condition imposed by having a circular geometry, the phase angle 
between neighboring blades around the annulus must sum to $2 \pi$. This interblade phase angle is expressed by the following equation.

$$
\sigma=\frac{2 \pi n}{N_{b}}
$$

Due to mistuning of the rotor blades, the interblade phase angles between the rotor blades and the vibration amplitudes of the rotor blades can be nonuniform around the rotor circumference (Kurkov [5]). This will generate multiple nodal diameters during flutter. Because the casing mounted transducers are in the stationary reference frame, it is necessary to construct a mathematical transformation of the pressure waves from the rotating reference frame to the stationary reference frame to properly interpret a spectral analysis of the pressure transducer data.

Consider an isolated rotor that is vibrating at frequency $\omega$ and a constant nodal diameter $n$. The vibration pattern of the blades in the rotating reference frame can be represented mathematically as

$$
e^{i\left(\omega t+n \theta_{r}\right)}
$$

The flow field will also respond in this fashion and any flow variable can be expressed as

$$
A e^{i\left(\omega t+n \theta_{r}\right)}
$$

where $A$ is the amplitude. The ampitude is a function of $\theta_{r}$ and is periodic with a period of $\frac{2 \pi}{N_{b}}$. Hence, the amplitude can be expressed as the Fourier series given below.

$$
A\left(\theta_{r}\right)=\sum_{m=-\infty}^{\infty} A_{m} e^{i m \theta_{r} N_{b}}
$$

$A_{m}$ is the coefficient of the $m t h$ Fourier spatial harmonic. Combining these last two relations yields the variation of any flow variable to the rotor blades vibrating at frequency $\omega$ with a constant nodal diameter $n$. Thus, the unsteady pressure wave for each nodal diameter can be represented by the Fourier series

$$
p_{n}=\sum_{m=-\infty}^{\infty} A_{m n} e^{i\left[\omega t+\left(n+m N_{b}\right) \theta_{r}\right]}
$$

Equation 4 was also presented previously by Jutras et al. [3]. To transform $\theta_{r}$ to the stationary reference frame, the following equation is used

$$
\theta_{r}=\theta+\Omega t-\beta
$$

where at time $t=0, \beta$ is taken as 0 . Substituting Eq. (5) into Eq. (4) gives the stationary reference frame representation of the flutter wave associated with nodal diameter $n$ as

$$
p_{n}=\sum_{m=-\infty}^{\infty} A_{m n} e^{i\left(\omega_{m n} t+k_{m n} \theta\right)}
$$

where

$$
\begin{gathered}
\omega_{m n}=\omega+k_{m n} \Omega \\
k_{m n}=n+m N_{b}
\end{gathered}
$$

The nodal diameter will be an integer value such that $-\frac{N_{b}}{2} \leqslant n \leqslant \frac{N_{b}}{2}$, for an even number of blades, which is the case in this investigation. For an odd number of blades, the range of nodal diameters is $-\frac{N_{b}-1}{2} \leqslant n \leqslant \frac{N_{b}-1}{2}$. The total unsteady pressure in the stationary reference frame is given in Eq. (8).

$$
p=\sum_{n=-\frac{N_{b}}{2}}^{\frac{N_{b}}{2}} p_{n}
$$

For a pressure wave having a frequency, $\omega$, and nodal diameter, $n$, in the rotating reference frame, Eq. (6) shows that the pressure wave will be comprised of several frequencies, $\omega_{m n}$, in the stationary reference frame. 
To help formulate the physical interpretation of Eq. (4), it is necessary to rearrange Eq. (4) into the form of Eq. (9).

$$
\begin{aligned}
& p_{n}=\sum_{m=-\infty}^{\infty} A_{n} e^{i\left[\omega t+n \theta_{r}\right]} A_{m} e^{i\left[m N_{b} \theta_{r}\right]} \\
& p_{n}=A_{n} e^{i\left[\omega t+n \theta_{r}\right]} \sum_{m=-\infty}^{\infty} A_{m} e^{i\left[m N_{b} \theta_{r}\right]}
\end{aligned}
$$

Equation (10) represents the total unsteady pressure in rotating reference frame coordinates after summing over all nodal diameters.

$$
p=\sum_{n=-\frac{N_{b}}{2}}^{\frac{N_{b}}{2}}\left[A_{n} e^{i\left[\omega t+n \theta_{r}\right]} \sum_{m=-\infty}^{\infty} A_{m} e^{i\left[m N_{b} \theta_{r}\right]}\right]
$$

Using Eq. (5) to transform Eq. (10) results in Eq. (11).

$$
p=\sum_{n=-\frac{N_{b}}{2}}^{\frac{N_{b}}{2}}\left[\begin{array}{l}
A_{n} e^{i[(\omega+n \Omega) t+n \theta]} * \\
\sum_{m=-\infty}^{\infty} A_{m} e^{i\left[m N_{b} \Omega t+m N_{b} \theta\right]}
\end{array}\right]
$$

The unsteady pressure distribution given by $\sum_{m=-\infty}^{\infty} A_{m} e^{i\left[m N_{b} \Omega t+m N_{b} \theta\right]}$ and the wave components given by $A_{n} e^{i[(\omega+n \Omega) t+n \theta]}$ are an integral part of each other. Equation (10) is used for transducers located in the rotating reference frame, i.e. blade mounted, and Equation (11) is used for transducers located in the stationary reference frame.

This analysis is applicable to all the types of flutter presented in Fig. 2, and can be used when the rotor is mistuned.

The relations above will now be applied to the case where there are two probes mounted in the casing at the same axial location, but at different circumferential locations.

\subsection{Two probe analysis}

To determine the nodal diameters associated with the flutter wave, it is necessary to perform a cross-power spectral analysis between two transducers, where both sets of data have the same time index. To compute the nodal diameter at a given frequency in the cross-power spectrum, the phase $(\phi)$ at that frequency is divided by the angular separation between the two transducers; i.e., there are two sets of data $p_{1}\left(t, \theta_{1}\right)$ and $p_{2}\left(t, \theta_{2}\right)$ from which the cross-power spectrum is computed. (The cross-power spectrum is computed by multiplying the Fourier transform of one transducer by the conjugate of the Fourier transform of the other transducer, $F T_{1} *$ conjugate $\left(F T_{2}\right)$.)

$$
\phi=n \Delta \theta
$$

or

$$
n=\frac{\phi}{\text { angular separation between transducers }}
$$

Moreover, this relation applies to transducer pairs that are in the rotating reference frame as well as those in the stationary reference frame. Once the nodal diameter has been determined, the frequency is calculated using Eq. (13).

$$
\omega_{m n}=\omega+k_{m n} \Omega
$$

In this paper, most frequencies have been non-dimensionalized by the once-per-revolution rotational frequency and are represented as engine orders, given by the symbol $E$.

$$
E=\frac{\text { frequency }}{\text { rotational frequency of the rotor }}
$$

The case where there are multiple probes mounted in the casing will now be considered. 
Table 1

Location of rotating coordinate system $\left(\theta_{r}\left(t_{o}\right)\right)$ with respect to stationary pressure transducer positions

\begin{tabular}{lccccc}
\hline Time & \multicolumn{5}{c}{ Pressure transducer \# } \\
\hline & 1 & 2 & $\ldots$ & 10 & 11 \\
$t=0$ & $\theta_{r, 1}$ & $\theta_{r, 2}$ & $\ldots$ & $\theta_{r, 10}$ & $\theta_{r, 11}$ \\
$t=\Delta t$ & $\theta_{r, 2}$ & $\theta_{r, 3}$ & $\ldots$ & $\theta_{r, 11}$ & $\theta_{r, 1}$ \\
$t=\Delta t * 2$ & $\theta_{r, 3}$ & $\theta_{r, 4}$ & $\ldots$ & $\theta_{r, 1}$ & $\theta_{r, 2}$ \\
$\ldots$ & $\ldots$ & $\ldots$ & $\ldots$ & $\ldots$ & $\ldots$ \\
\hline
\end{tabular}

\subsection{Multiprobe analysis}

Flutter is a traveling wave that extends around the circumference of the entire rotor. However, this may or may not be the case for flutter initiation. As shown by Kurkov [5], a single pair of casing mounted pressure transducers provides sufficient detail to study fully developed flutter. To better understand the initiation phase, a circumferential array of transducers at the same axial location upstream of the leading edge was used, as illustrated in Fig. 3. The multiprobe analysis outlined in this section provides the advantage of simultaneously capturing the flutter response about the entire circumference of the rotor. Although flutter initiation is not the focus of this paper, it is important to verify the validity and capabilities of the multiprobe methods presented in this section. There are 11 casing mounted pressure transducers that are equally spaced around the circumference of the rotor, see Fig. 3. This type of configuration meets the requirement of capturing the unsteady pressure field around the entire rotor at each instant in time. Furthermore, this configuration avoids the previously discussed problems associated with blade mounted transducers.

The circumferential distribution of the unsteady pressure field around the rotor allows the data to be investigated as if recorded in the rotating reference frame. There are two primary methods of investigating the data using the multiprobe analysis. The first is to analyze the unsteady pressure field at a given instant of time, where the pressure field is a function of $\theta_{r}, p\left(\theta_{r}\right)$. The second is to analyze the unsteady pressure field as a function of angular position and time, $p\left(\theta_{r}, t\right)$. Both of these methods can also be implemented in the stationary reference frame.

In the first method, which will be referred to as the 1D spatial method, a spectral analysis of the unsteady pressure field at time $t$ can be computed for $p\left(\theta_{r}, t\right)$. Note that the number of data points for this approach is equivalent to the number of casing mounted pressure transducers, 11 in this study. The spectral analysis of the circumferentially distributed transducers yields the spatial frequencies, which are the nodal diameters. Recall that nodal diameters are integers limited to the range of $-\frac{N_{b}}{2} \leqslant n \leqslant \frac{N_{b}}{2}$. Since the Fourier transform used in this investigation produced only positive frequencies, it is convenient to state the range of nodal diameters as $0 \leqslant n \leqslant N_{b}$. This fan has 22 blades, which means the possible nodal diameters are $0 \leqslant n \leqslant 22$. The Nyquist criteria requires 44 data points (i.e. pressure transducers) to avoid aliasing of the spatial frequencies, $n$. For this study, only 11 pressure transducers were available, meaning that aliasing will occur for any nodal diameter above 5 .

For the second method, which will be referred to as the two-dimensional space-time method, a spectral analysis of the unsteady pressure field can be computed for $p\left(\theta_{r}, t\right)$. For each pressure transducer, there is a recorded signal of pressure versus time, $p(t)$. Additionally, angular position versus time, $\theta_{r}(t)$, for each pressure transducer can be calculated using Eq. (5). Hence, for each pressure transducer $p\left(\theta_{r}, t\right)$ can be calculated. Due to the circular geometry of the fan, $\theta_{r}(t)$ must be between 0 and $2 \pi$ for each sample. When the data from each of the 11 equally spaced pressure transducers is combined correctly, a complete two-dimensional unsteady pressure field, which will be denoted as $\widetilde{p}\left(\theta_{r}, t\right)$, is calculated. Note that for any given time $t=t_{o}, \widetilde{p}\left(\theta_{r}, t\right)$ will have 11 pressure samples at angular positions between 0 and $2 \pi$. If the sampling frequency, $F_{s}$, is such that the time between samples is equal to the time it takes the blades to move from one pressure transducer to the next, the set of angular positions in $\theta_{r}\left(t_{o}\right)$ will be the same for all $t$, as illustrated in Table 1 .

From such a set of data a two-dimensional Fourier transform, FT2, can be computed. This approach quantifies the frequency and nodal diameter over a given time period.

\section{Results}

Results will now be presented for the fan encountering stall flutter along the $70 \%$ corrected speedline. The Two Probe Analysis and the Multiprobe Analysis techniques will be presented and compared with each other. Flutter was 

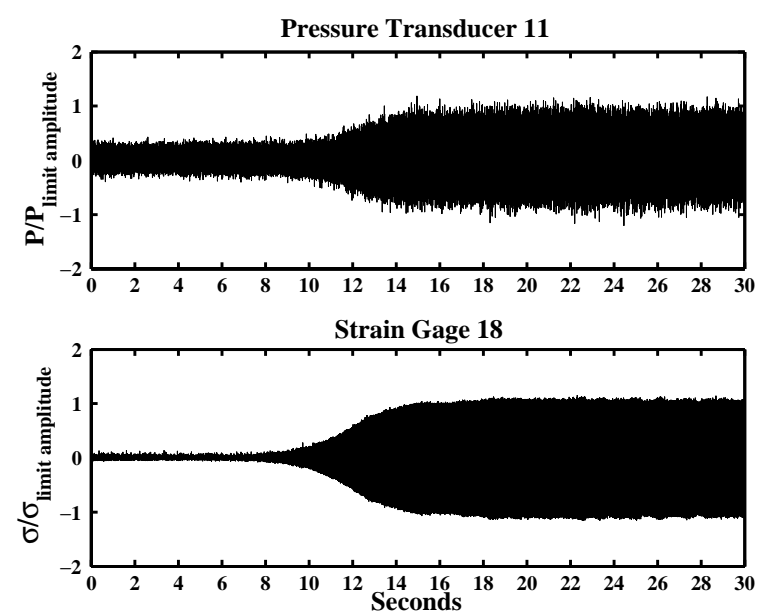

Fig. 4. Time dependent pressure and strain gage response for the fan as it is back-pressured into flutter.

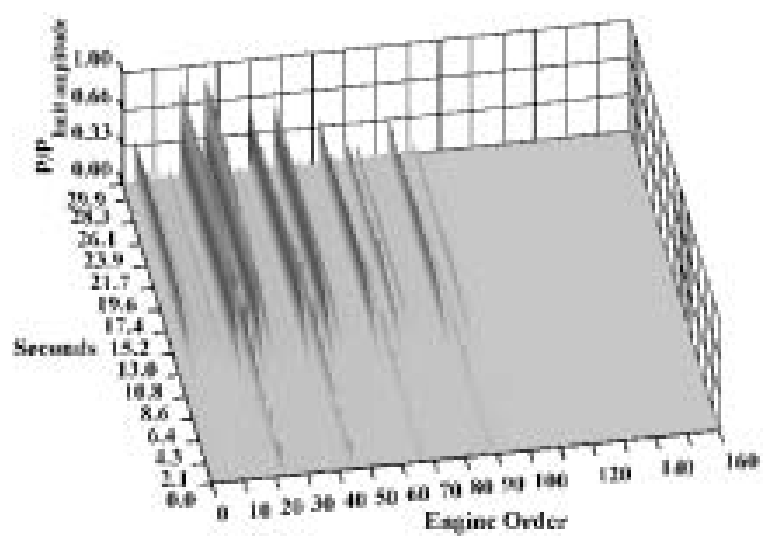

Fig. 5. Successive Fourier transforms of pressure transducer 11 as the fan is back-pressured into flutter.

generated by back-pressuring the fan using the throttling valves. Recording of the pressure transducers was initiated before flutter and continued well into the limit amplitude region. In the limit amplitude region the flutter vibration had reached a steady oscillatory motion of constant amplitude. The uncertainty in the pressure measurements had a maximum experimental error of $\pm 8 \%$.

\subsection{Two probe analysis}

Figure 4 shows the time signals recorded from pressure transducer 11 and strain gage 18. A Butterworth infinite impulse response filter of order 10 and cutoff frequency of $2 \mathrm{kHz}$ was applied to the pressure transducer signal to remove the pressure component due to blade passing for the purpose of comparing the two signals. Both transducers in Fig. 4 show a very similar response. Up to 8 seconds the pressure and strain gage signals have nearly constant amplitude. At approximately 8 seconds there is a distinct change in character of the pressure and strain gage signals. First, there is rapid growth of the pressure and strain, which is the classical response expected for an instability (flutter). At approximately 15 seconds the rapid growth region levels off into a steady oscillating response, which will be referred to as the limit amplitude region. The time dependent pressure measured in the stationary reference frame and the strain gage response measured in the rotating frame exhibit the same characteristics. This indicates that stationary reference frame pressure transducers can be used to quantify flutter initiation and limit amplitude response of blades for flutter monitoring. 


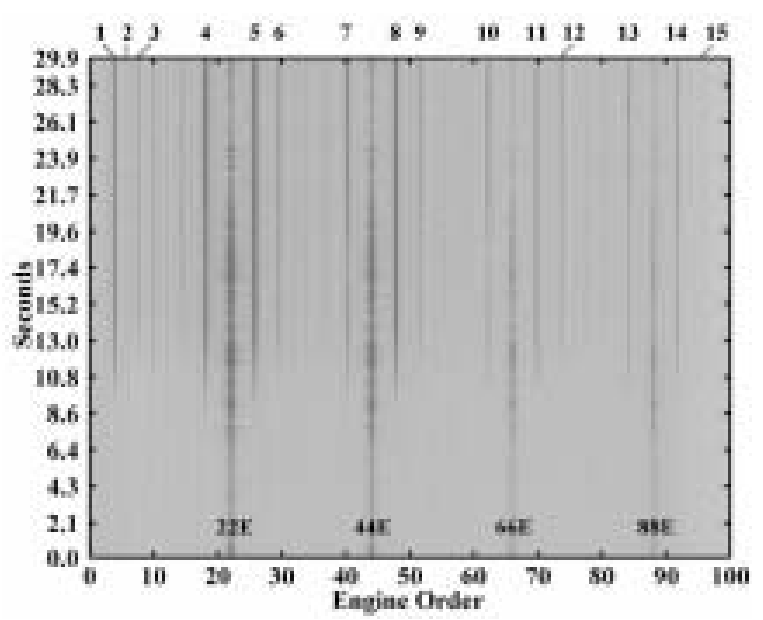

\begin{tabular}{cccc}
\hline label & $m$ & $f=411 \mathrm{~Hz}$ & $f=822 \mathrm{~Hz}$ \\
\hline 1 & 0 & $n=2$ & \\
2 & 0 & $n=4$ & \\
3 & 0 & $n=5$ & \\
4 & -1 & $n=2$ & \\
5 & +1 & $n=2$ & $n=4$ \\
6 & +1 & & \\
7 & -2 & $n=2$ & $n=4$ \\
8 & +2 & $n=2$ & \\
9 & +2 & & $n=4$ \\
10 & -3 & $n=2$ & \\
11 & +3 & $n=2$ & \\
12 & +3 & & \\
13 & -4 & $n=2$ & \\
14 & +4 & $n=2$ & \\
15 & +4 & &
\end{tabular}

Fig. 6. Top down view of Fig. 5 with the major wave harmonics and nodal diameters identified.

The pressure transducer and strain gage data were subdivided into smaller blocks of 8192 points. The frequency spectrum of each block was computed using the Fourier transform.

Figure 5 presents each of the FTs from pressure transducer 11 successively from the beginning to the end of the recorded data. By displaying the data in this manner the development of flutter can be visualized. The time from the first data point in each 8192-point data block is used for the time axis. The number of rotor rotations for 8192 points is 24. The blade passing frequency and its harmonics, integer multiples of 22E, have been reduced in amplitude to allow viewing of the flutter response. The dominant flutter responses occur in pairs about each blade passing harmonic. The primary flutter response frequencies have been labeled in Fig. 6, a top down view of Fig. 5. The signal-to-noise ratios of the $n=2$ responses at $m=-1$ and +1 are 42 and 47 respectively. The noise level was determined from the Fourier transform at 20 seconds, see Fig. 5. The signal-to-noise ratio was calculated by dividing the noise amplitude into the flutter response amplitude.

Consider Eq. (7) in the form

$$
f_{m n}=f+\left(n+m N_{b}\right) f_{\text {rot }}
$$

The rotational frequency $f_{\text {rot }}$ and the number of blades $N_{b}$ are known quantities from the experiment. The frequencies $f_{m n}$ are from the Fourier spectra, such as those in Fig. 5. The harmonic number $m$ is known by the grouping of the frequencies with the blade passing frequencies/harmonics. Leaving $n$ and $f$ unknown in Eq. (15). The nodal diameter $n$ can be calculated from cross-power spectra for each frequency $f_{m n}$, Eq. (12). The remaining unknown in Eq. (15) is the flutter frequency $f$.

Cross-power spectra were calculated from adjacent pairs of casing mounted pressure transducers. Equation (12) is used to calculate the nodal diameter for all the frequency responses in the cross-power spectra, except at the rotational frequencies of $22 \mathrm{E}, 44 \mathrm{E}, 66 \mathrm{E}, \ldots$. Figure 7 shows results for the $3.82 \mathrm{E}$ frequency line. The cross-power analysis was performed using each of the successive FTs of the individual transducers in pairs from the beginning to the end of the recorded data. Referring to Fig. 4, the rapid growth region of the flutter response begins somewhere around 8 seconds and continues through 15 seconds. From the 15 second mark to the end of the recorded data is the limit amplitude region of the flutter response. Figure 7 presents the normalized cross-power amplitude in the bottom plot with the corresponding calculated nodal diameter in the upper plot. This figure shows a fully established nodal diameter of 2 by the 8 second mark. It is interesting to note that there are some 2 nodal diameter responses at various points preceding 8 seconds during the transient into flutter. However, the signal-to-noise ratios are poor in this region. At 7.9 seconds the signal-to-noise ratio reaches 1.4.

Now that the nodal diameter is known for this frequency line of the cross-power spectrum, the flutter frequency can be quantified using Eq. (15). The flutter frequency was calculated to be $411 \mathrm{~Hz}$. This procedure was applied to all the frequency responses indicated in the cross-power spectra. Nodal diameters of 2, 4, and 5 were found for the $411 \mathrm{~Hz}$ flutter response with $m=0$, shown in Fig. 8. The amplitudes were normalized by the limit amplitude 

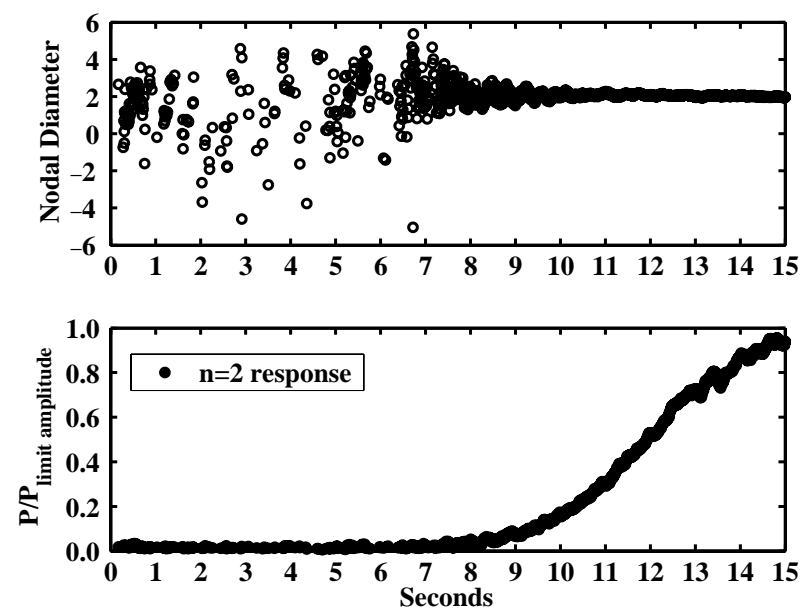

Fig. 7. Cross-power spectral analysis of pressure transducers 11 and 12 as the fan is back-pressured into flutter for $n=2$.

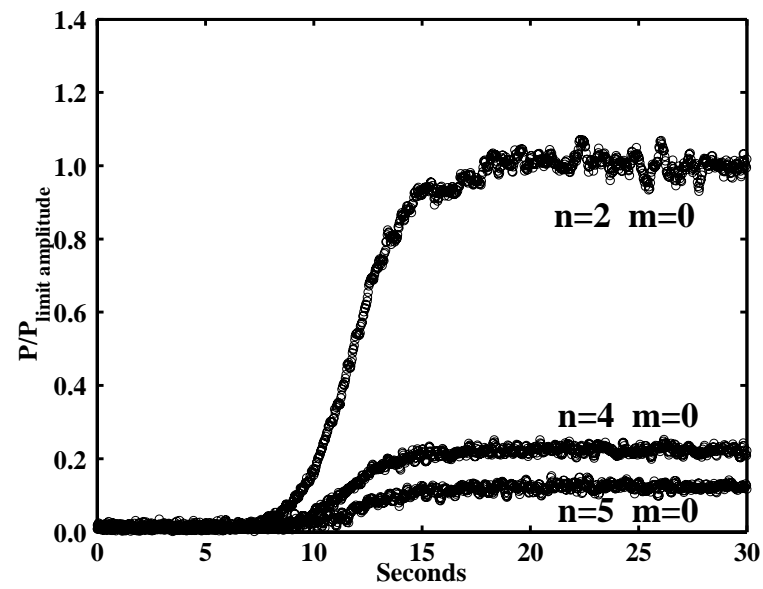

Fig. 8. Cross-power spectral analysis of pressure transducers 11 and 12 as the fan is back-pressured into flutter.

response at $\mathrm{n}=2$. Due to the rapid fall off in the other frequencies, see Figs 5 and 8 , it is believed that aliasing is not a significant issue in this analysis.

Interestingly the second harmonic of the flutter frequency was also identified in both the strain gage response and the pressure transducer response in flutter. Both frequencies are well below the Nyquist frequency of $37.5 \mathrm{kHz}$ for this experiment. At $411 \mathrm{~Hz}$, multiple harmonics of $m=0, \pm 1, \pm 2, \pm 3, \pm 4$, and \pm 5 were active for the nodal diameter of 2. A response was found for a nodal diameter of 4 at $822 \mathrm{~Hz}$ and harmonics of $m=1,2$, and 3. The largest responses occurred at $411 \mathrm{~Hz}$ for $n=2$ and $m= \pm 1$, followed by $n=2$ and $m= \pm 2$. The table in Fig. 6 summarizes the findings.

Data from strain gages 17 and 18 were analyzed in the same manner as the pressure transducer data. Figure 9 shows each of the FTs from strain gage 18 in a single waterfall plot. In a top down view of Fig. 9, individual nodal diameter and harmonic lines are shown in Fig. 10. Figures 9 and 10 show a primary frequency line at 1.82 engine orders. Cross-power spectra were calculated between strain gages 17 and 18. Figures 11 presents results from the cross-power analysis applied to the 1.82E frequency line. Both figures show an established nodal diameter of approximately 2 by the 8 second mark. In agreement with the pressure transducer analysis, results from the limit amplitude region of the strain gage data show a flutter frequency of $411 \mathrm{~Hz}$ at approximately 2 nodal diameters with a higher harmonic response at a frequency of $822 \mathrm{~Hz}$ and approximately 4 nodal diameters. Like with the pressure 


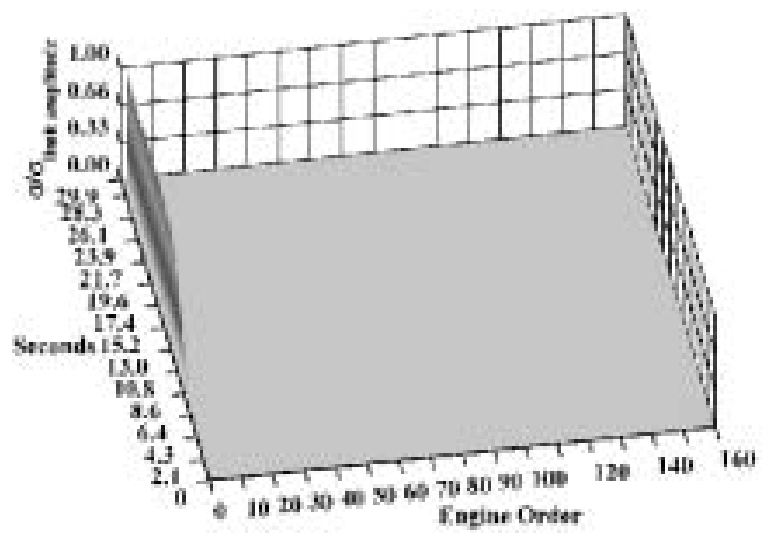

Fig. 9. Successive Fourier transforms from strain gage 17. The response occurs at 1.82 engine orders as the fan is back-pressured into flutter.

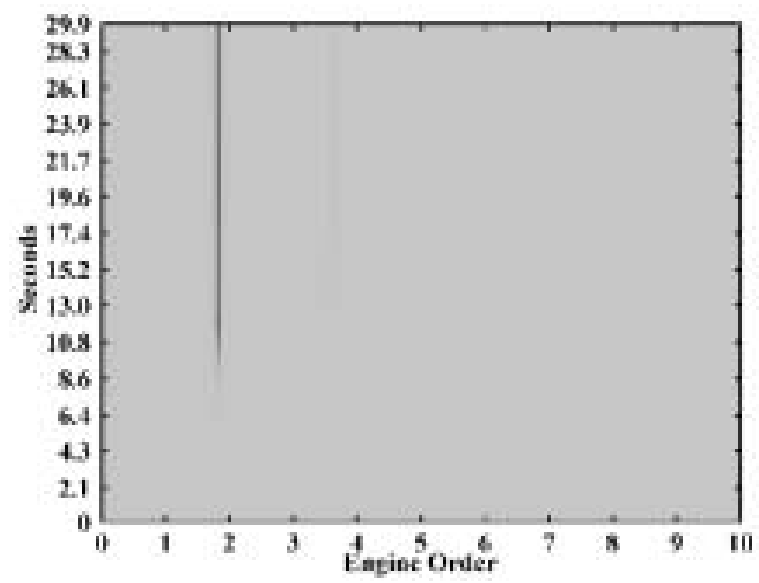

Fig. 10. Top down view of Fig. 9. The peak response occurs at 1.82 engine orders.

transducers, there are strong 2 nodal diameter responses at various points preceding 8 seconds during the transient into flutter.

The nodal diameter determined using the cross-power analysis was 2.34. As shown by the pressure transducers there are other nodal diameters present at the flutter frequency. For the stationary frame pressure transducers the different nodal diameters occur at different frequencies. However, the multiple nodal diameter response for the strain gages is occurring at a single frequency. Hence, the cross-power spectrum for the strain gages is giving a 'weighted average' for the nodal diameter, with a nodal diameter of 2 being the dominant response. Washburn [14] has also discussed this issue.

Figure 12 shows the remarkable similarity between a casing mounted pressure transducer and a blade mounted strain gage flutter response. In this figure, the pressure transducer flutter response is the $411 \mathrm{~Hz}$ frequency line for a nodal diameter of 2 and $m=0$ harmonic. The strain gage flutter response is the $411 \mathrm{~Hz}(1.82 E)$ frequency line. The correlation between the pressure transducers and strain gages is excellent.

\subsection{Multiprobe analysis}

Ideally all transducers, pressure or strain gages, function properly and the data has been recorded under optimal conditions, such as low noise. Unfortunately, in this investigation pressure transducer 13 malfunctioned at the start of testing, and pressure transducer 6 has considerable noise, see Fig. 13, which presents each pressure transducer's time dependent data during the transient into flutter. Without all the equally spaced pressure transducer data, the 

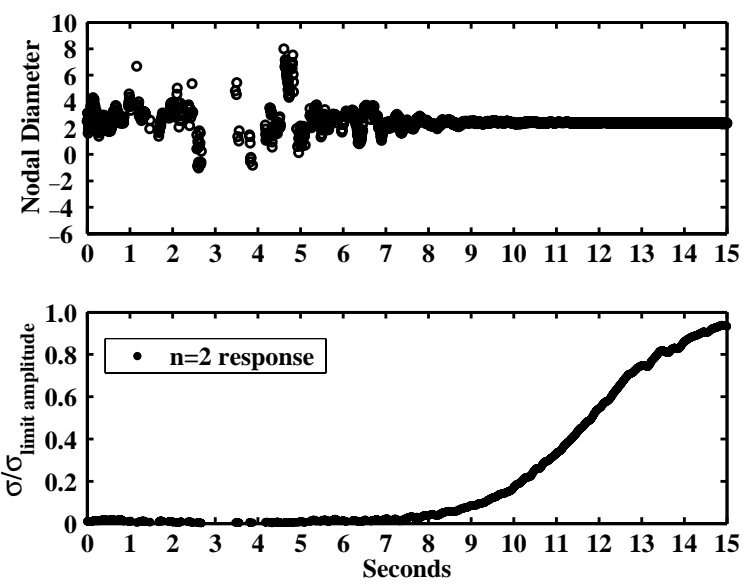

Fig. 11. Cross-power spectral analysis of strain gages 17 and 18 as the fan is back-pressured into flutter.

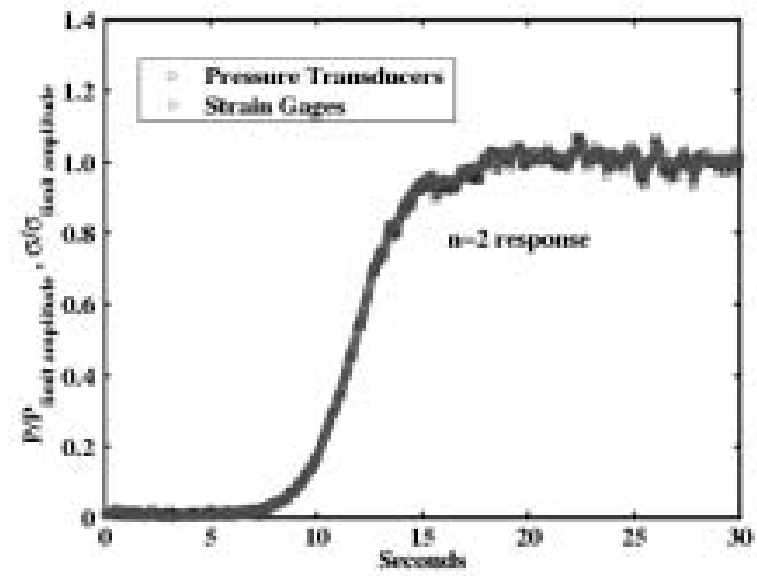

Fig. 12. Cross-power spectral analysis of strain gages 17 and 18 and pressure transducers 11 and 12 as the fan is back-pressured into flutter for $n=2$.

one-dimensional spatial and two-dimensional space-time methods can not be easily employed. The two-dimensional space-time method was still effective for analyzing the data. To compensate, Lagrange interpolation was used to estimate the missing data for pressure transducers 6 and 13, see the Appendix.

Before the pressure transducer data was transformed from the stationary reference frame to the rotating reference frame, each of the pressure transducer's data were digitally filtered to remove the blade passing frequency, and then re-sampled at the rotational frequency using linear interpolation. With the nine functional pressure transducers and the two interpolated pressure transducers, the unsteady pressure field $\widehat{p}\left(\theta_{r}, t\right)$ was created, as discussed in the Data Analysis section.

As with the two probe analysis, the unsteady pressure field, $\widehat{p}\left(\theta_{r}, t\right)$, was broken into smaller time blocks. The dimensions of the unsteady pressure blocks were 1024 points along the time axis by 11 points along the spatial axis. Two-dimensional Fourier transforms were calculated for each of the blocks. Figures 14 through 17 show surface plots of the Fourier transforms. The position of the data blocks analyzed and shown here start in the rapid growth region and move into the limit amplitude region. The flutter response at $411 \mathrm{~Hz}$ and 2 nodal diameters can easily be seen in Figs 14 through 17. The signal-to-noise ratio of the $n=2$ response at $411 \mathrm{~Hz}$ in Fig. 17 is 77 . Aside from the flutter frequency, responses at multiples of the rotational frequency are seen in Figs 14 through 17. In addition, the weaker $822 \mathrm{~Hz}$ response at a nodal diameter of 4 is evident in Figs 16 and 17. These results are in excellent 


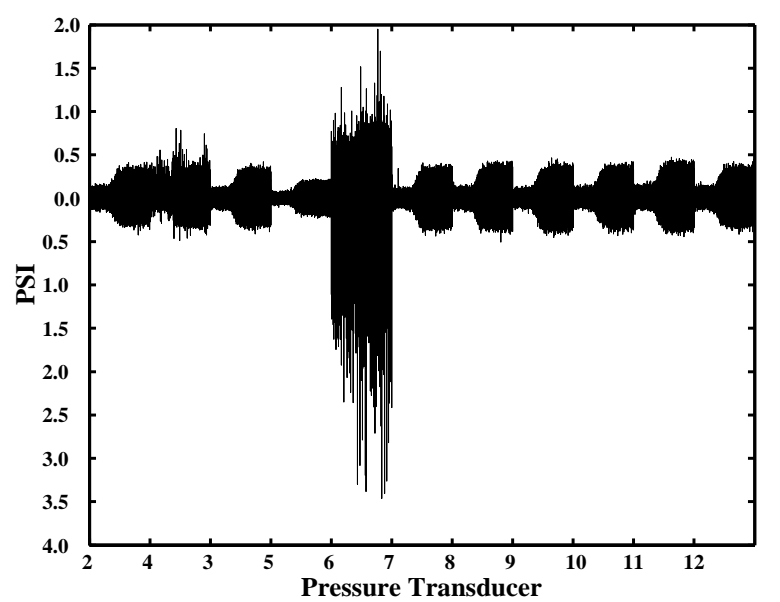

Fig. 13. A comparison of the pressure transducer data sets.

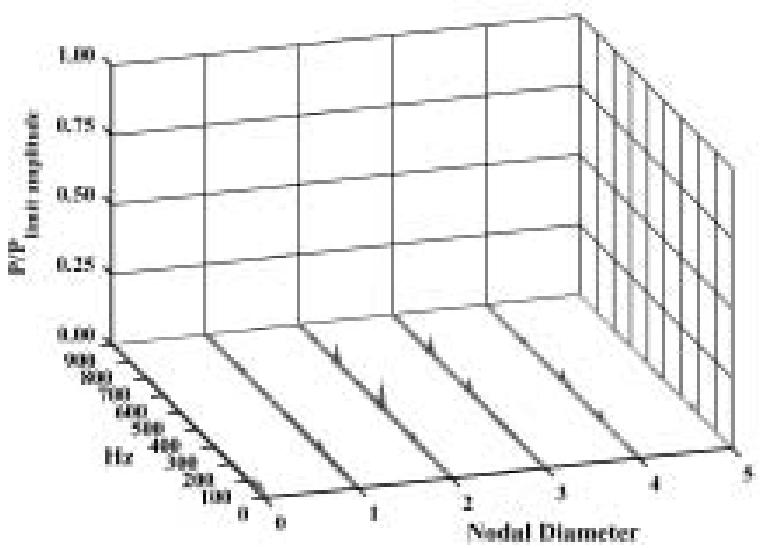

Fig. 14. Two-dimensional Fourier transform of pressure transducer data at 9 seconds.

agreement with those of the two probe analysis. The remaining significant responses are believed to be effects of using interpolation to estimate the missing pressure transducers, see Fig. 18.

\section{Conclusion}

A mathematical interpretation of the unsteady pressure field was developed and applied to a study of stall flutter in a single stage unshrouded axial flow fan. The analysis method employed consisted of two probe and multiprobe methods. Strain gage data from the rotating reference frame and high frequency response pressure transducer data from the stationary reference frame were analyzed. The stationary reference frame analysis technique involving the two probe method provides detailed spectral content about the nodal and harmonic makeup of the stall flutter instability. Likewise, it was shown that the typical two probe method applied to strain gage data provides a gross nodal diameter without any harmonic content information. The multiprobe method is applicable to both casing mounted high frequency response pressure transducers and blade mounted strain gages. The multiprobe method depends on the transducers being equally spaced around the circumference of the casing or rotor. In this study, one of the equally spaced pressure transducers failed. However, techniques were used to compensate for the missing pressure transducer which allowed the application of the two-dimensional space-time method to the pressure transducer data. 


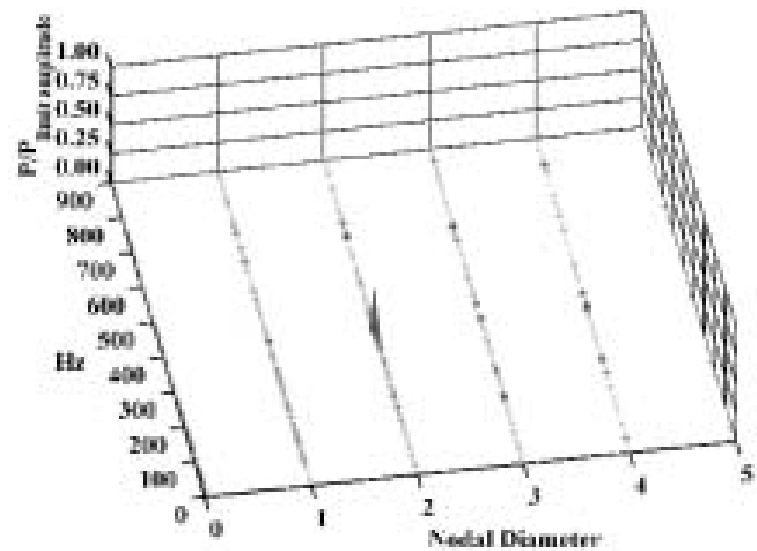

Fig. 15. Two-dimensional Fourier transform of pressure transducer data at 11 seconds.

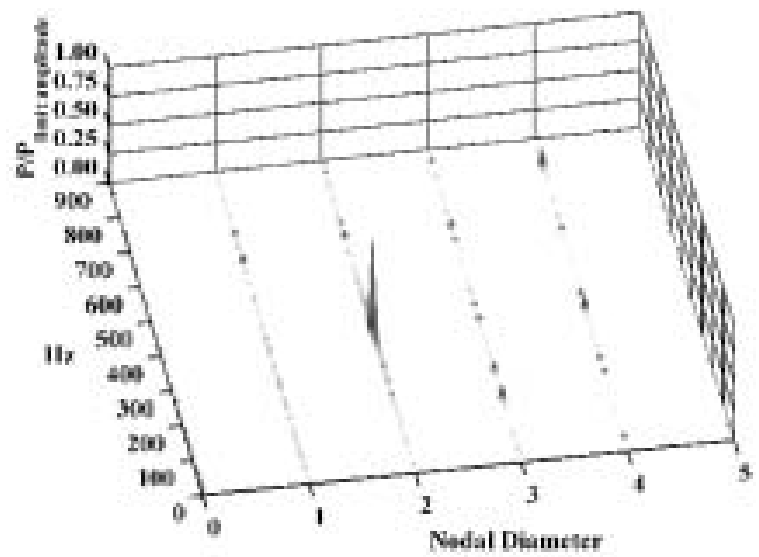

Fig. 16. Two-dimensional Fourier transform of pressure transducer data at 13 seconds.

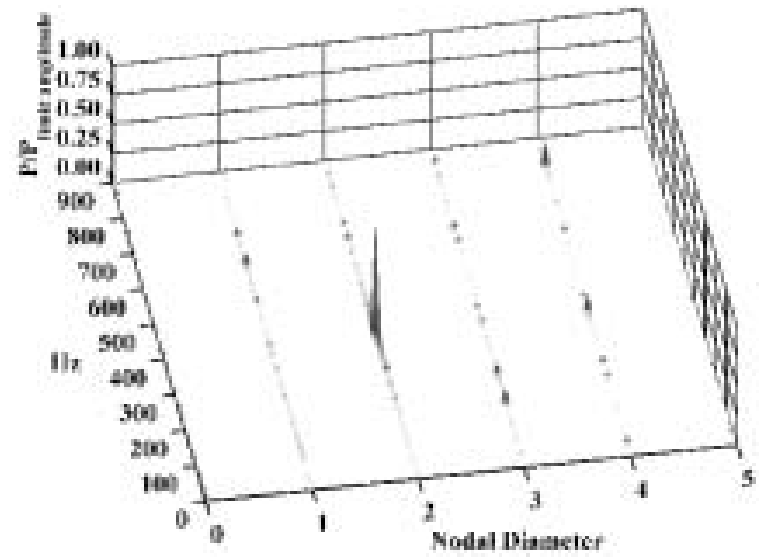

Fig. 17. Two-dimensional Fourier transform of pressure transducer data at 15 seconds.

The multiprobe method in its present form could not be applied to the strain gage data since they were not equally spaced. 


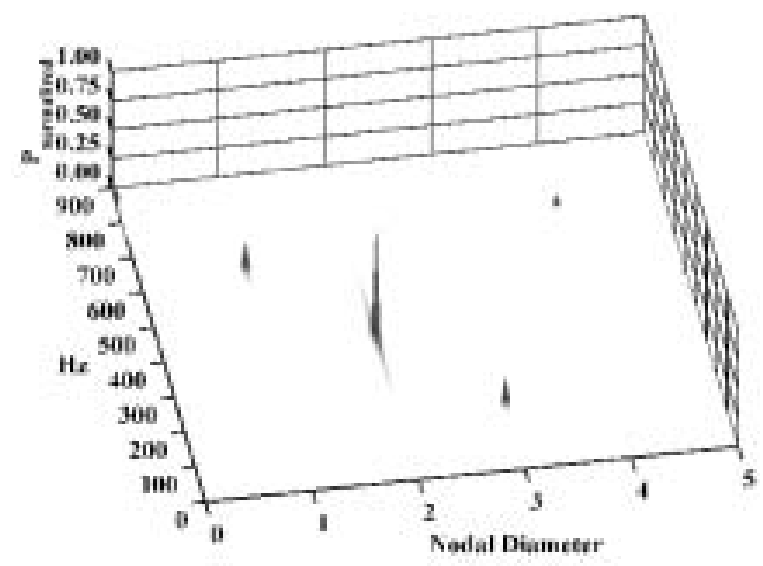

Fig. 18. Theoretical simulation of 2 nodal diameter flutter signal that includes the absence of two pressure transducers.

The two probe method was applied to the casing mounted high frequency response pressure transducer data and blade mounted strain gage data. The pressure transducer analysis produced results showing the stall flutter response to have multiple nodal diameter and multiple harmonic content. The flutter frequency of $411 \mathrm{~Hz}$ was identified with a nodal diameter of 2 . For the zeroth harmonic, $m=0$, nodal diameters of 2, 4 and 5 were identified for the flutter frequency of $411 \mathrm{~Hz}$. Additionally, the nodal diameter of 2 at $411 \mathrm{~Hz}$ showed responses at harmonics of $m=-5 \ldots 5$, with the largest amplitudes occurring at $m= \pm 1$. Responses at $822 \mathrm{~Hz}$, the second harmonic of the flutter frequency, were identified to have a nodal diameter of 4 at harmonics of $m=1,2$ and 3 .

The two probe method applied to the strain gage data provided results in agreement with the two probe pressure transducer results. Flutter responses at $411 \mathrm{~Hz}$ and $822 \mathrm{~Hz}$ were identified, where the gross nodal diameters were 2 and 4 , respectively.

For the two-dimensional space-time analysis a dominant flutter response at $411 \mathrm{~Hz}$ and a nodal diameter of 2 was identified. The secondary response at $822 \mathrm{~Hz}$ and nodal diameter of 4 was also identified. The existence of multiple nodal diameters is not strongly indicated in the figures because their relative amplitudes are much smaller than the $n=2$ response, however there is a response at $411 \mathrm{~Hz}$ and nodal diameters of 4 and 5.

\section{Appendix}

A theoretical analysis was conducted to test the effects produced when Lagrange interpolation is used to estimate the missing data for pressure transducers 6 and 13. Whether or not the interpolation method is acceptable requires a thorough analysis for each experiment. There are many factors to take into account, like the total number of transducers, the circumferential distance between each transducer, the location of the failed transducers and whether or not they are circumferentially next to each other, etc. To help ensure the interpolation method was valid for the present pressure transducer configuration, numerous tests to understand the effects of the interpolation method were conducted.

The theoretical analysis consisted of simulating the flutter for 11 pressure transducers as used in this experiment. The simulated data points for pressure transducers 6 and 13 were replaced with a constant value, 999,999. Transformation of the 11 pressure transducers is performed next. The transformed data, $\widehat{p}\left(\theta_{r}, t\right)$, is then searched for the constant value, 999,999, used for the missing pressure transducers, and then replaced by Lagrange interpolating from the neighboring points using a third order Lagrange polynomial. Figure 18 shows the results of such a test. The Lagrange interpolation created some random frequencies and nodal diameters. The simulated flutter response had a frequency of $411 \mathrm{~Hz}$ with a nodal diameter of 2 and is easily distinguished in the Fourier transform of Fig. 18. Several tests were conducted to add additional characteristics to the simulated data, such as re-sampling at a lower frequency. It was concluded that the two-dimensional space-time method could provide reliable results for data that was re-sampled to a lower frequency and when Lagrange interpolation is used to estimate the missing pressure transducers. 


\section{Acknowledgements}

The authors would like to gratefully acknowledge the support of this research by the Compressor Research Facility at Wright Patterson Air Force Base, Dayton, OH, Honeywell International, Inc., and Dr. A. Sanders.

\section{Nomenclature}

$A_{m n} \quad$ Pressure amplitude of $m$ th harmonic and $n$th nodal diameter

E $\quad$ Engine order

$f \quad$ Flutter frequency in rotating system, $\mathrm{Hz}$

$f_{m n} \quad$ Flutter frequency for $m$ th harmonic and $n$th nodal diameter in the stationary reference frame, $H z$

$f_{\text {rot }} \quad$ Rotational frequency, $\mathrm{Hz}$

$k_{m n} \quad$ Stationary reference frame mode number for $m$ th harmonic and $n$th nodal diameter

$m \quad$ Wave harmonic number

$N_{b} \quad$ Number of blades

$N_{c} \quad$ Corrected speed

$n \quad$ Nodal diameter

$p \quad$ Unsteady pressure

$p_{n} \quad$ Unsteady pressure for $n$th nodal diameter

$\widetilde{p} \quad$ Two-dimensional unsteady pressure field

$\widehat{p} \quad$ Interpolated two-dimensional unsteady pressure field

$\beta \quad$ Initial angle between rotating and stationary reference frames

$\theta \quad$ Angular position in stationary reference frame

$\theta_{r} \quad$ Angular position in rotating reference frame positive opposite rotation direction

$\sigma \quad$ Interblade phase angle

$\phi \quad$ Cross-power phase angle

$\Omega \quad$ Rotor speed, $\mathrm{rad} / \mathrm{sec}$

$\omega \quad$ Flutter frequency in rotating system, $\mathrm{rad} / \mathrm{sec}$

$\omega_{m n} \quad$ Flutter frequency for $m$ th harmonic and $n$th nodal diameter in the stationary reference frame, $\mathrm{rad} / \mathrm{sec}$

FT Fourier transform

FT2 Two dimensional Fourier transform

NSMS Noninterference Stress Measurement System

\section{References}

[1] J.A. Ekaterinaris and M.F. Platzer, Numerical Investigation of Stall Flutter, ASME Journal of Turbomachinery 118 (1996), $197-203$.

[2] R.B. Fost and P.S. Teets, Upgrading the Air Force Turbine Engine Research Center for Enhanced HCF Aeromechanics Evaluations with Light Probes, Third National Turbine Engine High Cycle Fatigue (HCF) Conference, 2-5 February, 1998.

[3] R.R. Jutras, R.B. Fost, R.M. Chi and B.F. Beacher, Subsonic/Transonic Stall Flutter Investigation of a Rotating Rig, NASA CR-174625, 1981.

[4] A.P. Kurkov, Measurement of Aerodynamic Work During Fan Flutter, Journal of Engineering for Power 105 (1983), $204-211$.

[5] A.P. Kurkov, Formulation of Blade Flutter Spectral Analyses in Stationary Reference Frame, NASA Technical Paper (1984), 2296.

[6] A.P. Kurkov, Measurements of Self-Excited Rotor-Blade Vibrations Using Optical Displacements, Journal of Engineering for Gas Turbines and Power 106 (1984), 44-49.

[7] A.P. Kurkov, Optical Measurement of Unducted Fan Blade Deflections, ASME Paper No. 89-GT-298, 1989.

[8] A.P. Kurkov and O. Mehmed, Optical Measurements of Unducted Fan Flutter, ASME Paper No. 91-GT-19, 1991.

[9] M.P. Mignolet and B. Choi, Robust Optimal Positioning of Strain Gauges on Blades, Proceedings of the 7th National Turbine Engine High Cycle Fatigue Conference, 2002.

[10] A.J. Sanders, K.K. Hassan and D.C. Rabe, Experimental and Numerical Study of Stall Flutter in a Transonic Low-Aspect Ratio Fan Blisk, ASME Paper GT2003-38355, 2003.

[11] L.D.G. Siden, Numerical Simulation of Unsteady Viscous Compressible Flows Applied to Blade Flutter Analysis, ASME Paper 91-GT-203, 1991.

[12] H. Stargardter, Optical Determination of Rotating Fan Blade Deflections, Journal of Engineering for Power 99(2) (1977), 204-209. 
[13] T.W. Swafford, D.H. Loe, D.L. Huff, D.H. Huddleston and T.S.R. Reddy, The Evolution of NPHASE: Euler/Navier-Stokes Computations of Unsteady Two-Dimensional Cascade Flow Fields, AIAA Paper No. AIAA-94-1834, 1994.

[14] R.S. Washburn, NSMS Nodal Diameter Analysis for Integral and Non-integral Vibratory Responses, Proceedings of the 7th National Turbine Engine High Cycle Fatigue Conference, 2002.

[15] S. Weber and M.F. Platzer, A Navier-Stokes Analysis of the Stall Flutter Characteristics of the Buffum Cascade, ASME Paper 2000-GT-385, 2000.

[16] J. Wu, D.L. Huff and L.N. Sankar, Evaluation of Three Turbulence Models for the Prediction of Steady and Unsteady Airloads, NASA Technical Memorandum 101413, 1989. 

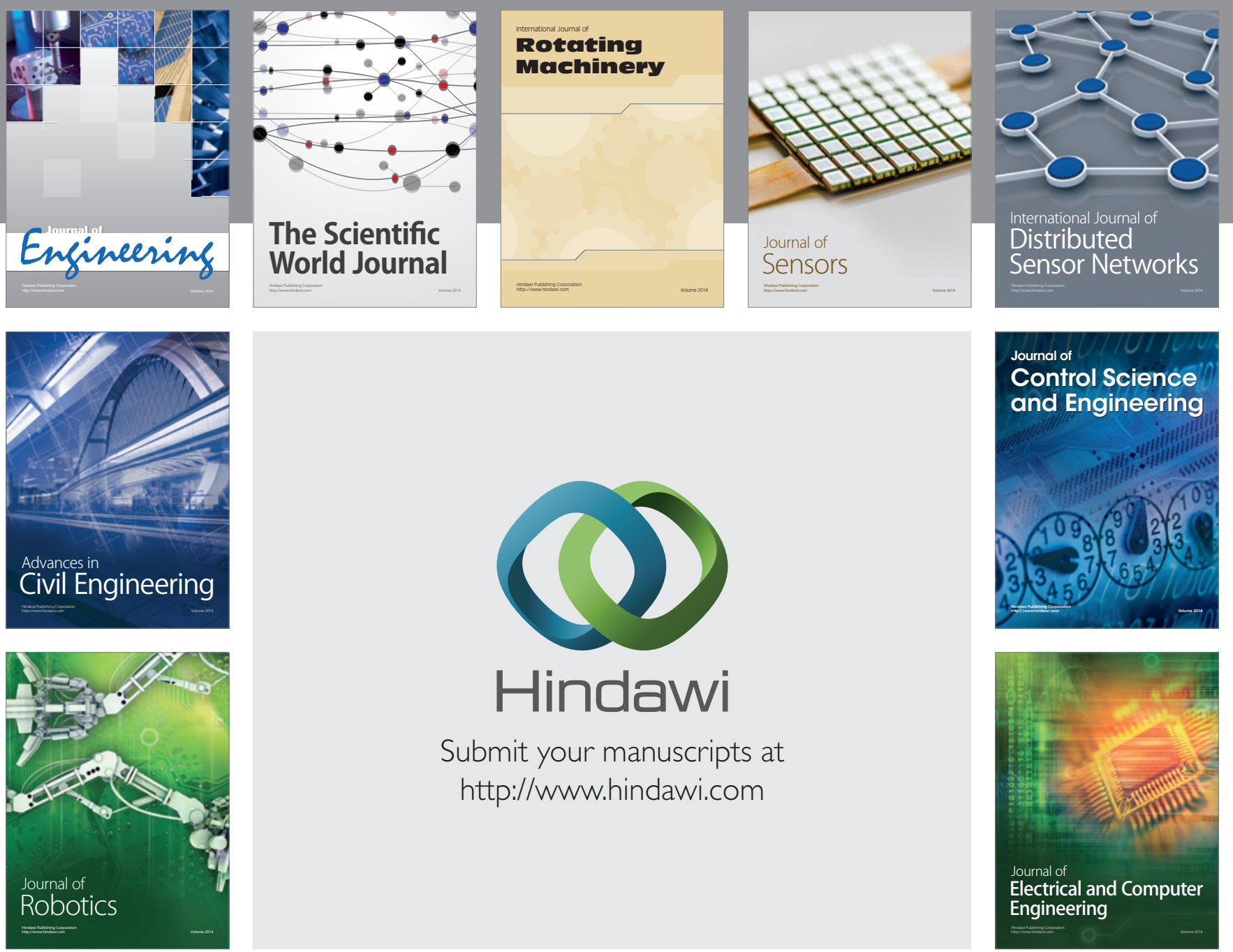

Submit your manuscripts at

http://www.hindawi.com
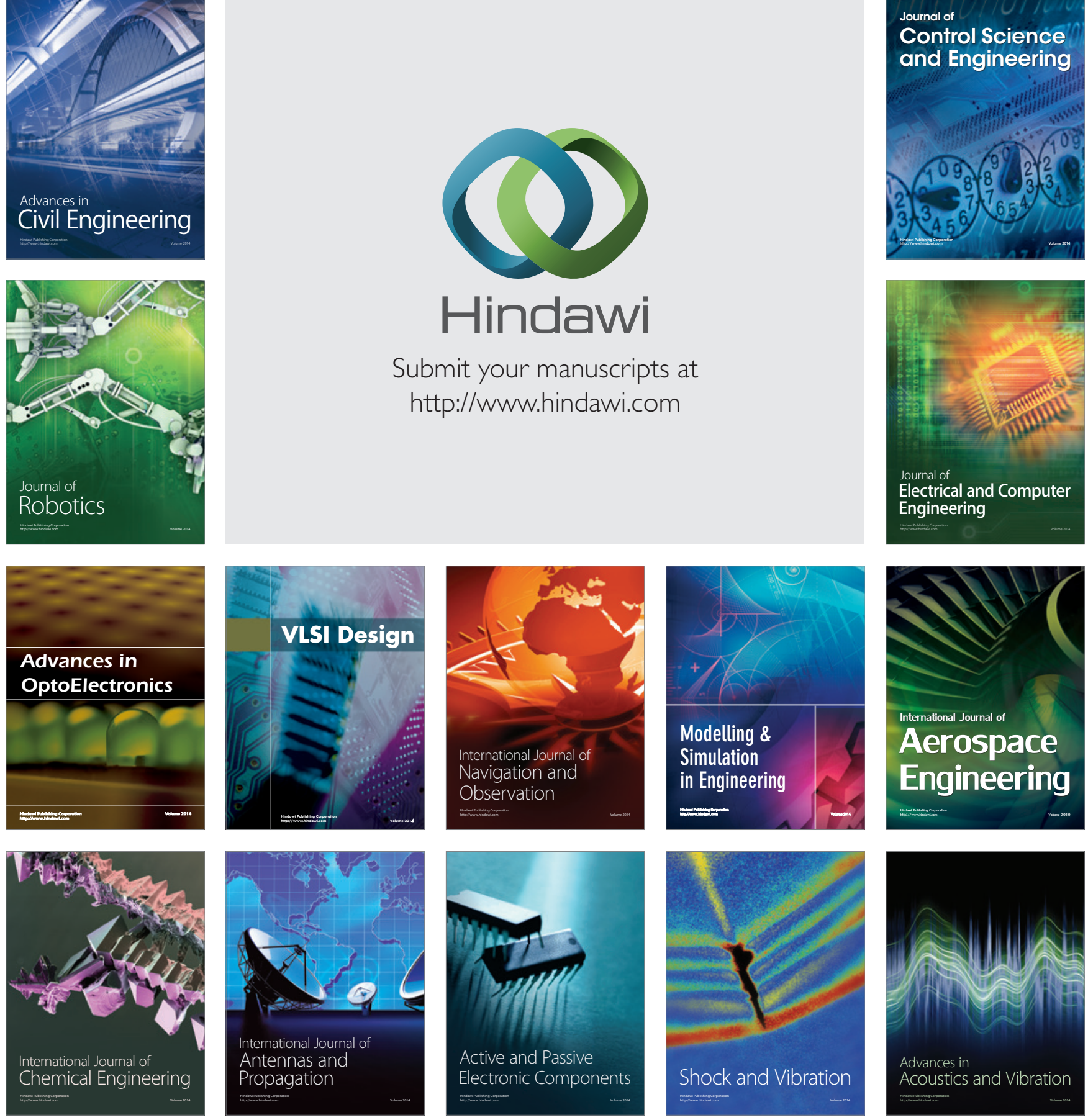